\title{
Clinical efficacy of acupoint application of Chinese herbal medicine to prevent acute exacerbation of stable chronic obstructive pulmonary disease: A study protocol for a randomized placebo-controlled trial
}

\section{Zhang Chuantao}

Chengdu University of Traditional Chinese Medicine

\section{Yang Hongjing}

Chengdu University of Traditional Chinese Medicine

\section{Gan Wenfan}

Chengdu University of Traditional Chinese Medicine

Xie Xiaohong

Chengdu University of Traditional Chinese Medicine

\section{Gao Peiyang}

Chengdu University of Traditional Chinese Medicine

Wu Jianying

Chengdu University of Traditional Chinese Medicine

Chen Jun

Chengdu University of Traditional Chinese Medicine

Zhao Keni

Chengdu University of Traditional Chinese Medicine

\section{Xiao Wei}

Chengdu University of Traditional Chinese Medicine

\section{Chen Keling}

Chengdu University of Traditional Chinese Medicine

Huang qingsong ( $\sim$ huangqingsong5802@163.com )

Chengdu University of Traditional Chinese Medicine https://orcid.org/0000-0003-1878-3579

\section{Study protocol}

Keywords: Chinese herb medicine, Acupoint application, Stable COPD, Acute exacerbation, Randomized controlled trial 
Posted Date: December 19th, 2019

DOI: https://doi.org/10.21203/rs.2.19290/v1

License: (c) (i) This work is licensed under a Creative Commons Attribution 4.0 International License. Read Full License 


\section{Abstract}

Background: Chronic obstructive pulmonary disease (COPD) is a major public health problem that severely affects the quality of life of patients and may even endanger their lives. The key to prevent the progression of COPD is active treatment during the stable phase that prevents and reduces acute exacerbation of COPD. Although modern medicine has achieved significant results in relieving the clinical manifestations of COPD, it is difficult to prevent its progression and acute exacerbation entirely. As one of the classic aspects of acupuncture and moxibustion therapy, acupoint application of Chinese herbal medicine (CHM) can improve the clinical efficacy of Western medicine in treating COPD. To date, however,there is no high-quality clinical trial to assess the effectiveness of $\mathrm{CHM}$ acupoint application directly in preventing acute exacerbation of stable COPD.Thus, we designed this randomized placebocontrolled clinical trial to evaluate the long-term efficacy and safety of $\mathrm{CHM}$ acupoint application in preventing the acute exacerbation of stable COPD.

Methods: The study is a randomized, double-blind, placebo-controlled trial, in which 200 stable COPD patients will be randomly and equally divided into the experimental group or control group. Both groups will undergo standard Western medicine treatment; however, the patients in the experimental group will be also treated with $\mathrm{CHM}$ acupoint application, while the control group will be given placebo acupoint application. The duration of the treatment will be 1 month and a follow-up for 11 months. The primary outcome will be the number of acute exacerbation episodes of COPD, and the secondary outcomes will include the lung function, St George's Respiratory Questionnaire, COPD Assessment Test, and 6-Minute Walk Test. A safety assessment will also be performed during the trial.

Discussion: The aim of this study is to evaluate the efficacy and safety of CHM acupoint application in preventing acute exacerbation of stable COPD. Our study will provide sound evidence to support the evidence-based medicine of $\mathrm{CHM}$ acupoint application as an additional measure in the prevention of acute exacerbation of stable COPD.

\section{Background}

Chronic obstructive pulmonary disease (COPD) is one of the leading causes of morbidity and mortality in the world ${ }^{1}$. It is estimated that 384 million people worldwide suffer from COPD ${ }^{2}$, of which China accounts for 100 million $^{3}$. In 2017, 3.2 million people died of COPD, a toll that is expected to reach 4.4 million per year by $2040^{1}$. Acute exacerbation episodes of COPD occur 0.5-3.5 times a year, which lead to further deterioration of airway injury, promotes the progression of the disease, and causes a huge economic burden on pateints ${ }^{2,4}$. Therefore, the key to COPD treatment is to treat it effectively during its stable period to prevent the occurrence of acute exacerbation.

Inhaled bronchodilators in stable COPD are central to symptom management. If necessary, triple therapy (inhaled glucocorticoids + long-acting $\beta 2$-agonist + long-acting muscarinic receptor antagonist) may be considered to reduce the deterioration frequency and increase labor tolerance. However, it cannot be 
confirmed if these drugs completely prevent disease progression and control COPD at different stages ${ }^{5}$. Moreover, these drugs have certain adverse reactions, such as palpitations, arrhythmia, and tremor, which limit the application of the drugs to a certain extent.

Acupuncture has been confirmed by some meta-analyses and clinical studies to safely and effectively treat patients with COPD and improve the exercise capacity as per the 6-Minute Walk Test and the quality of life of COPD patients as per the St George's respiratory questionnaire (SGRQ ${ }^{6-7}$. Acupoint application therapy is a compound treatment method that integrates meridians, acupuncture points, and herbs. First, the processed herbal ointment is placed on the medical adhesive medicine plaster. Then, some points are affixed with medicinal paste that has the characteristics of being non-traumatic, needing only small dose of medication, and having a direct effect on the acupuncture points. According to the basic Traditional Chinese Medicine (TCM) theory, acupuncture points application is similar to acupuncture in regulating meridians, yin and yang, and qi as well as the blood of the human body. Chinese medicines commonly used in acupoint application include Bai jiezi (Sinapis Semen), Ma huang (Ephedrae Herba), Xi xin (Asari Radix Et Rhizoma), Gan sui (Kansui Radix), Yan husuo (Corydalis Rhizoma), and Sheng jiang (Zingiberis Rhizoma Recens). A meta-analysis based on Chinese literature revealed that Bai jiezi, Xi xin, Gan sui, and Yan husuo, which were applied to acupoints (Feishu [BL 13], Dazhui [GV 14], and Danzhong [BL 43]) were considered as the basic medicines. Furthermore, those acupoints were considered as the basic acupoints to treat chronic respiratory diseases, such as asthma, COPD, chronic bronchitis, and allergic rhinitis. The therapeutic effect could be related to the anti-inflammatory effects of Bai jiezi and Gan sui ${ }^{8-9}$.

Acupoint application has been recorded in the Compendium of Materia Medica in detail. It is a common therapy for chronic pulmonary diseases, such as COPD, and it has been used by Chinese doctors for about 1300 years. Since the 1950s, acupoint application therapy has been widely carried out in 30 provinces, municipalities, and autonomous regions in China. A meta-analysis based on Chinese clinical trials ( 3,481 out of 32 randomized control trials [RCTs]) has shown that different combinations of Chinese herbal medicine (CHM) and acupoint application can improve the clinical efficacy of Western medicine in treating $\mathrm{COPD}^{10}$. To date, however, there is no high-quality clinical trial to assess the effectiveness of Chinese medicine acupoint application directly in preventing acute exacerbation of stable COPD. Therefore, we designed this clinical trial. To the best of our knowledge, this study is a rare, randomized, placebo-controlled design of RCT to evaluate the efficacy and safety of acupoint application of Chinese medicine in preventing the acute exacerbation of stable COPD.

\section{Methods/design}

\section{Study design and setting}

This study is a prospective, double-blind, randomized, placebo-controlled clinical trial. To prevent design bias, we will follow the Consolidated Standards of Reporting Trials statement ${ }^{11}$ and the Standard Protocol Items: Recommendations for Interventional Trials (SPIRIT) 2013 statement ${ }^{12}$. The study has been approved by the China Ethics Review Committee for Registered Clinical Trials (Approval No. 
ChiECRCT20190221). We registered the study in the Chinese Clinical Trial Registry, ChiCTR (Registration No. ChiCTR1900026564) in 2019. This trial will include 200 participants, and the participants will be informed of the benefits and risks of the study in detail. The written informed consent form will be included before the study, and the participants will be protected throughout the study. After obtaining written informed consent, eligible participants will be randomly assigned to the experimental group and control group in a ratio of 1:1. The entire study includes a screening assessment period, a 2-week run-in period, a 1-month treatment period, and an 11-month follow-up period. The schedule of enrollments, interventions, and assessments is shown in Figure 1 (the SPIRIT figure). The illustration of the design for clinical studies is presented below in Figure 2. The SPIRIT 2013 checklist is presented in Additional File 1.

\section{Participants}

This study will be conducted in the Hospital of Chengdu University of TCM. Participants will be recruited through a recommendation by the respiratory clinician and social media. All participants must meet the diagnostic criteria of Western medicine for COPD (Table 1) ${ }^{13}$ and the diagnostic criteria of TCM for lungspleen qi deficiency syndrome (Table 2$)^{14}$. The syndrome differentiation shall be determined independently by two designated deputy physicians of TCM.

\section{Eligibility criteria}

\section{Inclusion criteria:}

(1) Age range 45-70 years, male or female

(2) Grade 2-3 of the COPD diagnostic criteria in the Global Initiative for Chronic Obstructive Lung Disease (GOLD) guidelines ${ }^{13}: 20$ minutes after inhalation of salbutamol $400 \mu \mathrm{g}$, forced expiratory volume in 1 second/forced vital capacity $\left(\mathrm{FEV}_{1} / \mathrm{FVC}\right)<70 \%$ and $30 \%$ predicted value $\leq \mathrm{FEV}_{1}<80 \%$ predicted value

(3) Patient is in a stable phase of COPD with no respiratory infection and acute exacerbation of COPD in the past 4 weeks

(4) Patients with lung-spleen qi deficiency syndrome that meets the diagnostic criteria for common syndrome of TCM

(5) Participants volunteer to participate in the research and provide informed written consent

\section{Exclusion criteria:}

(1) Patients with a combination of chronic lung diseases, such as bronchial asthma, interstitial lung disease, active tuberculosis, or bronchiectasis, requiring intervention or treatment

(2) Patients with a combination of severe primary diseases, such as severe mental disorders, cardiovascular and cerebrovascular diseases, liver and kidney diseases, endocrine diseases, hematopoietic system diseases, malignant tumors, etc 
(3) Presence of skin wounds, skin ulcers, and skin infections on the application site

(4) Patients allergic to CHM, excipients, or dressings used in this project

(5) Patients with limited limb movements

(6) Women who are breastfeeding, pregnant, or preparing for pregnancy

(7) Patients participated in any other clinical studies in the past 6 months

\section{Randomization, allocation concealment}

A statistician, the member of the Sichuan TCM evidence-based Medicine Center will generate 200 random serial numbers using the SAS 9.2 Software (SAS. Cary, USA), and stratified randomization will be based on the GOLD classification of COPD. Eligible patients will be randomized into the experimental or control groups in a 1:1 ratio. The group numbers will be provided in a continuous manner in sealed envelopes made from carbonless paper. The envelopes will be kept by a study administrator who will not directly participate in the recruitment or follow-up of any participant. The administrator will open one envelope and provide the participant with their group number on the day of inclusion. Until the completion of the trial, the researchers will not know the grouping of the participants.

\section{Blinding}

The placebo paste used in this study is similar to the CHM paste of the trial group in shape, smell, and color. Acupoint application therapists and patients will be blinded to the treatment allocation throughout the course of the study. Only in emergencies, such as serious adverse events (AE), or if the patient needs emergency treatment, can the researchers report to the principal researcher to decide whether to expose the blind. Once the participant is exposed, the case will be treated as an exfoliated case and will not be included in the efficacy analysis. However, if there are adverse reactions, these should be included in the AEs analysis and detailed information about exposure will be recorded in the "early exit page of participants" of the case report form (CRF), including the time, cause, treatment, and treatment status of blind-exposed participants.

\section{Interventions}

Both groups will be treated with standard Western medicine. The treatment plan is based on the GOLD ${ }^{13}$ and the Guidelines for the Diagnosis and Treatment of Chronic Obstructive Pulmonary Disease (Revised 2013 $)^{15}$. All participants will be taught to use inhaled agents correctly, and they would have to quit smoking and prevent catching colds. Drug selection (based on the patient's pulmonary function) and symptoms and risk of acute exacerbation will be stratified, including that of GOLD grade 2 - regular inhalation of tiotropium bromide powder for inhalation (Si Li Hua, Boehringer Ingelheim Pharma GmbH \& amp; Co.KG [Germany], $18 \mu \mathrm{g} * 30$ capsules), $18 \mu \mathrm{g}$ per inhalation, once a day; and GOLD grade 3 - regular use of budesonide and formoterol fumarate powder for inhalation (Symbicort Turbuhaler, AstraZeneca 
AB [Sweden], $160 \mu \mathrm{g} / 4.5 \mu \mathrm{g} /$ inhalation, 60 inhalations), $164.5 \mu \mathrm{g}$ per inhalation, twice a day. The experimental group will be treated with acupoint application of $\mathrm{CHM}$ at the same time, while the control group will be treated with acupoint application of placebo. The course of treatment is 1 month, and the course of follow-up will be once every 3 months for 11 months. The TCM and acupoint selection program is based on academic literature data ${ }^{16-17}$ and the "Guidelines for the Clinical Application of Acupoint Application of Winter Diseases and Acupuncture Points (Draft)"18. The placebo used in the control group is made of buckwheat flour without any herbs, but has added honey and ginger juice to maintain an appearance similar to that used in the experimental group (Figure $3 \mathrm{c}$ and $\mathrm{d}$ ), thus making the plaster used in the two groups indistinguishable for patients and therapists. The standard operating procedure (SOP) for acupoint application is as follows:

1. Prepare the herbal materials: Bai jiezi (Sinapis Semen), Ma huang (Ephedrae Herba), Huang qi(Astragali Radix), Ban xia (Pinelliae Rhizoma), Xi xin (Asari Radix Et Rhizoma), and Gan sui (Kansui Radix) are mixed in a dose ratio of 3:3:2:1:1:2. The raw materials are ground into fine powder (diameter $<75 \mu \mathrm{m}$ ) by a special powder mixer of $\mathrm{CHM}$.

2. Add the liquid base: Put the mixed herbal powder into the container, add ginger juice and honey (1:2), and mix well to make a thick herbal ointment.

3. Add the sticky plaster: Take $10 \mathrm{~g}$ ointment and place it evenly on the center of the specially made medical sticky plaster.

4. Places of application: Feishu (BL13, bilateral), Dingchuan (EX-B1, bilateral), Gaohuang (BL43, bilateral), Zusanli (St36, bilateral), Dazhui (GV14), and Danzhong (CV17)

5. Treatment procedures: To find the acupuncture points, first disinfect the application site with iodophor routinely, and then stick the plaster on the acupuncture points. The time of each application is 3-4 hours, depending on the individual reaction of each patient. We considered the patient's personal constitution and tolerance, and usually the patient can tolerate the plaster. If patients consciously presented any discomfort, such as obvious burning sensation, severe itching, or blisters, they would be advised to immediately remove the ointment application and deal with it accordingly. The treatment will be performed twice a week for 4 weeks, total 8 times.

In this study, we will take the following measures to control the quality of acupoint application: (1) the TCM ointment and placebo ointment will be produced by Sichuan Green Medicine Technology Development Co., Ltd. (Sichuan, China), in line with the regulatory guidance requirements issued by the China Food and Drug Administration, (2) the location of acupoints will be determined by the doctors trained in acupuncture, and (3) the data and results will be monitored by the Sichuan TCM evidencebased Medicine Center.

\section{Outcome measurement}

The primary outcome includes the number of acute exacerbation episodes of COPD. COPD exacerbation is defined as an acute worsening of respiratory symptoms resulting in additional therapy ${ }^{19}$. 
Secondary outcome measures include:

1. FEV1 and FEV1/FVC change values of pulmonary function measurement

2. Changes in the SGRQ score

3. Changes of the COPD Assessment Test score

4. 6-Minute Walk Test

\section{Criteria for stopping treatment}

Participants will be informed that they have the right to discontinue treatment and withdraw from the research project for any reason at any time, and the reason for withdrawal will be recorded in the CRF. If they quit, they will receive standardized treatment. The criteria for stopping treatment and withdrawal from research projects are as follows: (1) There is severe allergy to the research dressings and drugs during the treatment, and the patient cannot continue being a part of the clinical research. (2) Participant's compliance is poor, or other drugs prohibited by this research scheme are being consumed. (3) Voluntary withdrawal from the trial or loss of follow-up.

\section{Compliance}

In this study, certain measures will be taken to ensure the compliance of the participants. Before entering the group, the participants will be carefully screened to understand their history of previous drug use, detailed information on the test content, course of treatment, adverse reactions of the treatment, and some possible burdens on the participants. Furthermore, written informed consent of the participants will be obtained. During the experiment, the professional and service quality of medical personnel should be maintained at optimum to create a good doctor-patient relationship. It is also important to establish healthy communication channels in time. For example, a contact card can be created to contact the participant, or record the telephone number in the record book. Two to three days before each follow-up visit, the researcher can call the participant to remind them of the last time they were interviewed. If the participants have difficulty getting to the hospital, a shuttle bus can be arranged.

\section{Observation and treatment of adverse events}

The dosage of CHM used in this study is within the recommended range of the Pharmacopoeia of the People's Republic of China (2015 edition), and all operating procedures will strictly follow the standard operating guidelines. In this study, patients could be allergic to research drugs or dressings during the application period, such as rash, skin itching, skin redness and swelling, or skin burning. We will strictly adhere to the criteria and avoid including those who are allergic to TCM or the accessories/dressings used in this study. In the course of the trial, it is necessary to determine whether the patients have related AEs due to the application. Moreover, laboratory tests will be performed to analyze blood, urine, feces, electrocardiogram, and kidneys and liver function, before and after treatment. Individuals reporting mild and moderate AEs will be treated for symptoms and closely observed. If serious AEs occur, the patients will be admitted to the hospital immediately for systematic treatment and reported to the principal 
researcher and ethics committee within 24 hours. All details of relevant and unexpected AEs, such as the time, severity, and suspicious causes of the AEs, will be recorded in detail in the CRF.

\section{Sample size estimation}

This is a parallel, randomized controlled study. The experimental group will be treated with acupoint application of $\mathrm{CHM}$, and the control group with placebo acupoint application. The number of acute attacks of COPD will be observed as the main outcome index within a year. According to previous literature reports ${ }^{20}$, it is estimated that the number of acute exacerbation episodes of COPD in the control group is 2.29 , with a standard deviation of about 1.23. After acupoint application of $\mathrm{CHM}$, the number of attacks in the experimental group is 1.64 times, with $\alpha=0.05$ (bilateral) and $\beta=0.10$. The sample size was 76 each in the experimental group and the control group and was calculated using the PASS 11 software. With an estimated dropout rate of $20 \%$, a total of 190 patients is enrolled. In the actual study, 100 cases will be included in each group.

\section{Data management and quality control}

The researchers in the trial team will be required to attend a training workshop before the trial begins. Each one will receive a copy of the trial protocol, and they will be asked to adhere to the protocol throughout the study. Researchers should fill in clinical trial records in a timely, accurate, complete, standardized, and true manner to ensure that all CRFs are accurate and consistent with the original data. Data management should be carried out according to the SOP of data management, and data entry and sorting will be done using EpiData 3.1 software. In order to ensure the reliability and accuracy of the data, two database operators will independently enter the same data, then conduct data consistency test, and proofread it multiple times. Files are stored in a secure and accessible manner. Security measures will be managed by user identification codes and passwords, and monthly backups are stored on CD media. Finally, the database is locked and analyzed according to the protocol, and confirmed and reviewed by the main researchers.

\section{Statistical analysis}

We will use the Statistical Package for the Social Sciences (SPSS version 21.0) statistical software for analysis, and use subgroup analysis methods to explain the efficacy and safety of acupoint sticking in different grades of GOLD patients to obtain comprehensive information. The measurement data will be expressed as mean \pm standard deviation, which is consistent with the normal distribution. The pairing ttest will be used before and after treatment. The t-test will be used for comparison between groups (homogeneity test of variance was performed, 0.05 is used as the test standard). For those which do not conform to the normal distribution or whose variance is not uniform, the rank sum test will be applied instead. The count data will be analyzed by chi-square test, and the grade data will be tested by rank sum test. All tests will be performed on both sides, and $\mathrm{P}<0.05$ will be statistically significant. 
A full analysis set (FAS, including completed trials, shedding cases and excluded cases) will be used for baseline data, and the FAS set and per-protocol (PP) set will be used for efficacy evaluation (PP set, including completed cases, and excluding shedding cases and rejection cases). If the two analytical conclusions are consistent, the credibility of the test results can be enhanced. If not, their differences should be fully discussed and explained. The missing data should be replaced by the method of sequence mean value in a FAS.

\section{Discussion}

COPD is the most common chronic respiratory disease. In China, COPD shows the "four high" characteristics of high morbidity, high disability rate, high mortality rate, and high disease burden, and it has become one of the most prominent public health and medical problems ${ }^{21}$. Although modern medicine has a good effect in relieving the symptoms of COPD, these measures still cannot fully control the disease progression and prevent acute exacerbation ${ }^{22}$. There is still a need to continue to develop new interventions to improve efficacy. CHM acupoint application is a kind of pulmonary rehabilitation method based on the theory of TCM, which applies CHM to certain acupuncture points of the human body to treat and prevent diseases. It is one of the suitable techniques of TCM, designated and popularized by the administration of TCM. It has a wide range of population usage and social influence in China. Although there have been many clinical reports on the intervention of $\mathrm{CHM}$ acupoint application in the treatment of COPD, there are still many problems in the design of these studies ${ }^{23}$. The use of random methods is not rigorous, and there is no description of blinding and allocation hiding. Additionally, the correct sample calculation method was not offered, AEs were not recorded in detail, and the intention-to-treat (ITT) analysis not performed on the shedding and missing cases is prone to bias risk. Therefore, we designed this placebo-controlled RCT to evaluate the efficacy and safety of CHM acupoint application in preventing acute exacerbation of stable COPD.

Compared with previous studies, we focus on assessing the effects of acupoint application on the longterm efficacy of stable COPD (future acute exacerbation) and patient activity tolerance. However, it cannot be ignored that this study also has certain limitations. First, in clinical practice, pure COPD patients are rare, and most patients present with other diseases such as cor pulmonale, hypertension, diabetes, and coronary heart disease. Therefore, the practicability (extrapolation) of the results in this study is not clear and needs to be further evaluated. Second, the study is being performed in Sichuan, China, and it is uncertain whether the relative effects of the trial drugs would be similar in other ethnic groups. Despite the limitations, we believe that this study will be helpful in finding the long-term benefits of $\mathrm{CHM}$ acupoint application in the prevention of acute exacerbation of stable COPD. In the future, multicenter RCTs should be conducted and multi-dimensional comparison should be conducted.

\section{Declarations}

\section{Ethical approval}


The research program has been approved by the China Ethics Review Committee for Registered Clinical Trials (Approval No. ChiECRCT20190221), and the members of the ethics committee tracked the design and implementation of the research. Prior to randomization, all individuals will be explicitly explained that the trial involves two types of interventions, and that only those who agree to sign the informed consent and voluntary participation in the trial will be included in the study.

\section{Trial status}

This paper is based on protocol version 2.0 dated 3 November 2019. Recruitment began in December 2019 , and approximate date of completion is December 2020. Any major protocol changes will be notified to the ethics committee and updated on the Chinese Clinical Trial Registry.

\section{Additional file}

Additional file 1: SPIRIT 2013 checklist. (DOC $139 \mathrm{~kb}$ )

\section{Acknowledgements}

We are grateful to the Sichuan Science and Technology Program for funding this study. We also would like to thank Editage (www.editage.cn) for English language editing.

\section{Funding}

This study is supported by Sichuan Science and Technology Program (18ZDYF0922). The funder don't take part in the study design, data collection and analysis, or the preparation of the manuscript. The funder has provided only financial support for the study.

\section{Ethics approval and consent to participate}

The study is in compliance with the Declaration of Helsinki (Edinburgh 2000 version). The final amendments (version 2.0: November 3, 2019) and the consent form have been reviewed and approved by the China Ethics Committee of Registering Clinical Trials (reference number: ChiECRCT20190221). If there is any amendment to the protocol, approval must be again sought from the Ethics Committee. Written informed consent will be obtained from each participant before enrolment.

\section{Consent for publication}

Not applicable

\section{Availability of data and materials}

We declared that materials described in the manuscript, including all relevant raw data, will be freely available to any scientist wishing to use them for non-commercial purposes, without breaching participant confidentiality. 


\section{Competing interests}

The authors declare that they have no competing interests.

\section{Authors' contributions}

ZCT and HQS contributed to the design of the study protocol. YHJ and GWF drafted the manuscript. ZCT, GPY, and YHJ revised the manuscript prior to final approval. XXH, WJY, ZKN, XW, and CKL will conduct the study. HQS wil be responsible for the overall management of the study. All authors have read and approved the final manuscript.

\section{Authors' information (optional)}

1 Department of Respiratory Medicine, Hospital of Chengdu University of Traditional Chinese Medicine, Chengdu 610072, China

2 Department of Critical Care Medicine, Hospital of Chengdu University of Traditional Chinese Medicine, Chengdu 610072, China

3 Department of Gastroenterology, Hospital of Chengdu University of Traditional Chinese Medicine, Chengdu 610072, China

\section{Abbreviations}

AE: Adverse event; COPD: Chronic obstructive pulmonary disease; $\mathrm{CHM}$ : Chinese herbal medicine; CAT: COPD Assessment Test; CONSORT: Consolidated Standards of Reporting Trials; CRF: Case report form; $\mathrm{FEV}_{1}$ : Forced expiratory volume in 1 second; FVC: Forced vital capacity; FAS: full analysis set; GOLD: Global Initiative for Chronic Obstructive Lung Disease; PP set: per-protocol set; RCT: Randomized controlled trial; SGRQ: St George's Respiratory Questionnaire; SOP: Standard operation procedure; SPSS: Statistical package for social science; SAS: statistical analysis system; SPIRIT: Standard Protocol Items: Recommendations for Interventional Trials; SOP: Standard operating procedure; TCM: Traditional Chinese Medicine; 6MWT: 6-Minute Walk Test

\section{References}

1. Adeloye D, Chua S, Lee C, Basquill C, Papana A, Theodoratou E, et al. Global and regional estimates of COPD prevalence: Systematic review and meta-analysis. J Glob Health 2015; 5(2):020415.

2. GA R, et al. Global, regional, and national age-sex-specific mortality for 282 causes of death in 195 countries and territories, 1980-2017: a systematic analysis for the Global Burden of Disease Study 2017. Lancet 2018; 392(10159):1736-1788.

3. Wang $\mathrm{C}$, et al. Prevalence and risk factors of chronic obstructive pulmonary disease in China (the China Pulmonary Health [CPH] study): a national cross-sectional study. Lancet 2018; 
391(10131):1706-1717.

4. Donaldson G C, et al. Impact of Prolonged Exacerbation Recovery in Chronic Obstructive Pulmonary Disease. Am J Respir Crit Care Med 2015; 192(8):943-950.

5. Braido F, et al. Commentary on "Costs of pharmacotherapy of chronic obstructive pulmonary disease in relation to changing Global Initiative for Chronic Obstructive Lung Disease guidelines $(2007,2011$, and 2017 updates)". Pol Arch Intern Med. 2019; 129(5):299-300.

6. Wang J, et al. Acupuncture Therapy for Functional Effects and Quality of Life in COPD Patients: A Systematic Review and Meta-Analysis. Biomed Res Int 2018; 2018:3026726.

7. Feng J, et al. Acupuncture for chronic obstructive pulmonary disease (COPD): A multicenter, randomized, sham-controlled trial. Medicine (Baltimore) 2016; 95(40):e4879.

8. Xian Y F, et al. Comparison of the anti-inflammatory effects of Sinapis alba and Brassica juncea in mouse models of inflammation. Phytomedicine 2018; 50:196-204.

9. Zhang J S, et al. Anti-inflammatory Ingenane Diterpenoids from the Roots of Euphorbia kansui. Planta Med. 2018; 84(18):1334-1339.

10. Li F, et al. Effect of point application on chronic obstructive pulmonary disease in stationary phase and effects on pulmonary function: a systematic evaluation of randomized controlled trials. J Tradit Chin Med 2012; 32(4):502-514.

11. Schulz K F, et al. CONSORT 2010 Statement: updated guidelines for reporting parallel group randomized trials. Open Med 2010; 4(1):e60-8.

12. Chan A W, et al. SPIRIT 2013 statement: defining standard protocol items for clinical trials. Ann. Intern. Med. 2013; 158(3):200-207.

13. Singh D, et al. Global Strategy for the Diagnosis, Management, and Prevention of Chronic Obstructive Lung Disease: the GOLD science committee report 2019. Eur. Respir. J. 2019; 53(5).

14. Chinese Society of Traditional Chinese Medicine Internal Medicine Branch Pulmonary Diseases Professional Committee. Guidelines for TCM Diagnosis and Treatment of Chronic Obstructive Pulmonary Disease (2011 Edition). Journal of Traditional Chinese Medicine 2012; 53(1):82.

15. Chinese Medical Association Respiratory Diseases Society Chronic Obstructive Pulmonary Diseases Group. Guidelines for the diagnosis and treatment of chronic obstructive pulmonary disease (revised edition 2013). Chinese Journal of Tuberculosis and Respiratory 2013; 36(4):255-264.

16. Fang $Y \mathrm{G}$, et al. A study on the basic drugs and points for point application in summer to treat the diseases with attacks in winter. J Tradit Chin Med 2010; 30(3):180-184.

17. An P, et al. Preliminary study on acupoint application and selection of acupoints in stable stage of chronic obstructive pulmonary disease. Chinese and foreign medical treatment 2019; 06:170-174.

18. Chinese Acupuncture Association. A Guidelines for Clinical Application of "Acupoint Application for Winter Disease and Summer Treatment" (Draft). Chinese Acupuncture 2009; 07:541-542.

19. Wedzicha J A, et al. COPD exacerbations: defining their cause and prevention. Lancet 2007; 370(9589):786-796. 
20. Zhang YW, et al. Therapeutic effect of acupoint application on stable phase of chronic obstructive pulmonary disease. Shanghai Journal of Acupuncture and Moxibustion 2016; 35(09):1065-1069.

21. Zhang XE, et al. Research Progress on Epidemiology and Economic Burden of Chronic Obstructive Pulmonary Disease. Chinese Journal of Prevention and Control of Chronic Diseases 2019; 25(6):472476.

22. Murray C J, et al. Global, regional, and national disability-adjusted life years (DALYs) for 306 diseases and injuries and healthy life expectancy (HALE) for 188 countries, 1990-2013: quantifying the epidemiological transition. Lancet 2015; 386(10009):2145-2191.

23. Yu JJ , et al. A Meta-analysis of point application on pulmonary rehabilitation in patients with stable chronic obstructive pulmonary disease. Clinical Journal of Chinese Medicine 2018; 10(25):1-5.

\title{
Tables
}

Table 1. Western medicine diagnostic criteria for chronic obstructive pulmonary disease (COPD)
Symptoms Two or more symptoms such as chronic cough, sputum expectoration, wheezing, chest stuffiness, lack of energy, anorexia, cyanosis, shortness of breath (especially during physical activities), muscular atrophy, unintended weight loss (in later stages), cyanosis, etc.

Risk Age, gene polymorphism, exposure to tobacco smoke, occupational exposure factors to dusts and chemicals, exposure to fumes from burning fuel, socio-economic status, people with asthma and smoking habits, HIV infection, etc.

Spirometry Required to establish diagnosis: The presence of a post-bronchodilator $\mathrm{FEV}_{1} / \mathrm{FVC}<0.7$ confirms the existence of persistent airflow limitation, and thus COPD.

\begin{abstract}
FEV 1 /FVC: forced expiratory volume in 1 second/forced vital capacity.COPD:Chronic obstructive pulmonary disease. HIV:Human immunodeficiency virus.
\end{abstract}

Table 2. Diagnostic criteria for Traditional Chinese Medicine (TCM) differentiation of lungspleen qi deficiency syndrome 
4 Cough or wheezing, shortness of breath, and aggravation of movement

૫ Tiredness, fatigue, or spontaneous sweating, and aggravation of movement

4 Fear of the wind, easy to catch a cold

․ Loss of appetite, reduced eating

․ Epigastric distension or abdominal distension or loose stool

4 The tongue body fat or has the tooth mark, the tongue coating thin white or white greasy, the pulse sink is fine or sinks slowly or is thin and weak

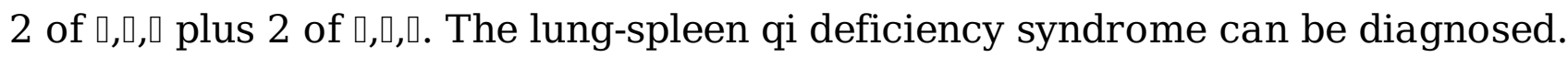

\section{Figures}

\begin{tabular}{|c|c|c|c|c|c|c|c|c|c|c|}
\hline & \multicolumn{10}{|c|}{ STUDY PERIOD } \\
\hline & \multirow{2}{*}{$\begin{array}{c}\begin{array}{c}\text { Enrolment } \\
\text { (weeks) }\end{array} \\
-2 \text { to }-1\end{array}$} & \multirow{2}{*}{$\begin{array}{c}\begin{array}{c}\text { Allocation } \\
\text { (weeks) }\end{array} \\
0\end{array}$} & \multicolumn{4}{|c|}{$\begin{array}{l}\text { Treatment } \\
\text { (weeks) }\end{array}$} & \multicolumn{4}{|c|}{$\begin{array}{l}\text { Follow-up } \\
\text { (weeks) }\end{array}$} \\
\hline Timepoint & & & 1 & 2 & 3 & 4 & 16 & 28 & 40 & 52 \\
\hline Visit & & & & & & 1 & 2 & 3 & 4 & 5 \\
\hline \multicolumn{11}{|l|}{ ENROLMENT } \\
\hline Eligibility screen & $\checkmark$ & & & & & & & & & \\
\hline Informed consent & $\checkmark$ & & & & & & & & & \\
\hline Allocation & & $\sqrt{ }$ & & & & & & & & \\
\hline \multicolumn{11}{|l|}{ INTERVENTIONS } \\
\hline \multicolumn{11}{|l|}{$\begin{array}{l}\text { Acupoint application of } \\
\text { Chinese herbal } \\
\text { medicine + Western } \\
\text { medicine treatment }\end{array}$} \\
\hline \multicolumn{11}{|l|}{$\begin{array}{l}\text { Placebo acupoint } \\
\text { application + Western } \\
\text { medicine treatment }\end{array}$} \\
\hline \multicolumn{11}{|l|}{ ASSESSMENTS } \\
\hline $\begin{array}{l}\text { Number of acute } \\
\text { aggravation }\end{array}$ & & $\checkmark$ & & & & $\sqrt{ }$ & $\checkmark$ & $\checkmark$ & $\checkmark$ & $\checkmark$ \\
\hline Lung function test " & & $\checkmark$ & & & & $\sqrt{ }$ & $\sqrt{ }$ & $\checkmark$ & $\checkmark$ & $\sqrt{ }$ \\
\hline SGRQ score & & $\checkmark$ & & & & $\sqrt{ }$ & $\checkmark$ & $\checkmark$ & $\sqrt{ }$ & $\checkmark$ \\
\hline CAT questionnaire & & $\checkmark$ & & & & $\sqrt{ }$ & $\checkmark$ & $\sqrt{ }$ & $\sqrt{ }$ & $\sqrt{v}$ \\
\hline 6MWT & & $\checkmark$ & & & & $\checkmark$ & $\sqrt{ }$ & $\checkmark$ & $\checkmark$ & $\sqrt{ }$ \\
\hline \multicolumn{11}{|l|}{ SAFETY ASSESSMENTS } \\
\hline Skin reaction & & $\checkmark$ & $\checkmark$ & $\checkmark$ & $\checkmark$ & $\checkmark$ & $\checkmark$ & & & \\
\hline Laboratory tests ${ }^{[2]}$ & & $\checkmark$ & $\checkmark$ & $\checkmark$ & $\checkmark$ & $\sqrt{ }$ & $\checkmark$ & & & \\
\hline
\end{tabular}




\section{Figure 1}

SPIRIT figure: Schedule of enrollments, interventions, and assessments [1] Lung function tests: FEV1, forced expiratory volume in one second; FVC, forced volume capacity [2] Laboratory tests: blood, urine, feces, electrocardiogram, and kidney and liver function tests SGRQ: St George's Respiratory Questionnaire; CAT:COPD Assessment Test; 6MWT: 6-Minute Walk Test

\section{Figure 2}

Illustration of the design for clinical studies

要

\section{Figure 3}

Illustration of the sticky plaster (a) appearance of the acupoint plaster from the front; (b) appearance of the acupoint plaster from the back; (c) Chinese herbal paste on the plaster; (d) Buckwheat plaster; both (c) and $(\mathrm{d})$ are identical in appearance

\section{Supplementary Files}

This is a list of supplementary files associated with this preprint. Click to download.

- Additionalfile1.doc 